\title{
Forward Collision Warning with a Single Camera
}

\author{
Erez Dagan \\ MobileEye Vision \\ Technologies Ltd. \\ Jerusalem, Israel \\ erez.dagan@mobileye.com
}

\author{
Ofer Mano \\ MobileEye Vision \\ Technologies Ltd. \\ Jerusalem, Israel \\ ofer.mano@mobileye.com
}

\author{
Gideon P. Stein \\ MobileEye Vision \\ Technologies Ltd. \\ Jerusalem, Israel \\ gideon.stein@mobileye.com
}

\author{
Amnon Shashua \\ Hebrew University \\ Jerusalem, Israel \\ shashua@cs.huji.ac.il
}

\begin{abstract}
The large number of rear end collisions due to driver inattention has been identified as a major automotive safety issue. Even a short advance warning can significantly reduce the number and severity of the collisions. This paper describes a vision based Forward Collision Warning $(F C W)$ system for highway safety. The algorithm described in this paper computes time to contact (TTC) and possible collision course directly from the size and position of the vehicles in the image - which are the natural measurements for a vision based system - without having to compute a $3 D$ representation of the scene. The use of a single low cost image sensor results in an affordable system which is simple to install. The system has been implemented on real-time hardware and has been test driven on highways. Collision avoidance tests have also been performed on test tracks.
\end{abstract}

\section{Introduction}

One of the major challenges of the next generation of road transportation vehicles is to increase the safety of the passengers and of pedestrians. Over 10 million people are injured yearly worldwide in road accidents. These include two to three million severely injured and 400,000 fatalities. The financial damage of accidents is estimates as 1$3 \%$ of world GDP. Rear-end collisions constitute a significant proportion of the total accidents (29.5\% in USA and $28 \%$ in Germany)[1].

Lack of attention by the driver is identified as the cause for $91 \%$ of driver related accidents. According to a 1992 study by Daimler-Benz (cited in [1]), if car drivers have a 0.5 -second additional warning time, about $60 \%$ of rear-end collisions can be prevented. An extra second of warning time can prevent about $90 \%$ of rear-end collisions.

This places Forward Collision Warning (FCW) high on the list of solutions that can contribute significantly to reduction of the number and the severity of driving accidents. A range sensor mounted on the vehicle could provide a prac- tical solution to this problem [3]. However, the prices of the traditional systems available today (typically based on Radar sensors) and their limited performance (narrow field of view and poor lateral resolution) have prevented such systems from entering the market. From a technological point of view, fusion of radar and vision is an attractive approach. In such a system the radar gives accurate range and range-rate measurements while vision solves the angular accuracy problem of radar. However this solution is costly.

This paper presents MobilEye's vision based FCW system including experimental results. The algorithm described in this paper computes the Time-to-Contact (TTC) and possible collision course directly from the size and position of the vehicle in the image - which are the natural measurements for a vision based system - without having to compute a 3D representation of the scene. In particular, accurate range measurements are not required. Measurements from the host vehicle (speedometer, gas-pedal or brakepedal position etc.) are not required but can be used in the complete system as a secondary filter to further anticipate the drivers intentions and reduce unnecessary alarms.

\subsection{The MobilEye Advance Warning System (AWS)}

Using a single forward facing camera located typically near the rear view mirror, the MobilEye-AWS detects and tracks vehicles on the road ahead providing range, relative speed and lane position data. The system detects also the lane markings and road edges and measures the distance of the host vehicle to road boundaries. Thus it combines together on the same platform Forward Collision Warning, Lane Departure Warning and Headway Monitoring. It can also be connected to active safety systems.

The camera used in this work has VGA sensor $(640 \times 480)$ and a horizontal FOV of $47^{\circ}$. A small display unit and a pair of left and right speakers (see Figure 1) inside the car provide audio and visual warnings, allowing the driver to 


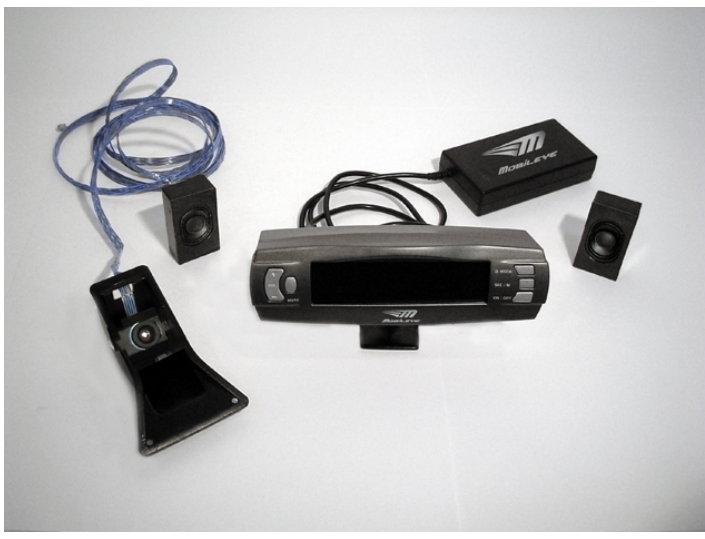

(a)system components

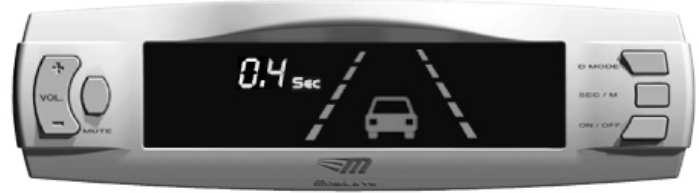

(b)display panel

Figure 1: The MobilEye-AWS.

react to various types of dangerous situations and to reduce the risk of accidents. The system warning thresholds are adaptable to different driving styles.

\section{Momentary Time to Contact}

One method for FCW analyzed in [4] uses time to contact (TTC) to trigger the warning. A Forward Collision Warning (FCW) is issued when the time-to-contact (TTC) is lower than a certain threshold - typically 2 seconds. We will define the momentary Time To Contact $\left(T_{m}\right)$ as

$$
T_{m}=-\frac{Z}{V}
$$

where $Z$ is the relative distance to the target and $V$ the relative velocity.

Since distance and relative speed are not natural vision measures, we will show that we can represent the momentary TTC as a function of scale-change in the image in a given sampling interval $(\Delta t)$. This value can be computed accurately from the image sequence as shown in [2].

The perspective projection model of the camera gives:

$$
w_{t}=\frac{f W}{Z_{t}}
$$

where $w_{t}$ is the width of the target in the image at time $t$, $Z_{t}$ is the distance to the target, $W$ is the vehicle width, and $f$ is the camera focal length.
We define scale-change $S$ as the ratio between the width in the image in two consecutive frames

$$
S=\frac{w_{1}}{w_{0}}=\frac{\frac{f W}{Z_{1}}}{\frac{f W}{Z_{0}}}=\frac{Z_{0}}{Z_{1}}
$$

When the time interval between $Z_{1}$ and $Z_{0}$ is small we can write:

$$
Z_{1}=Z_{0}+V \Delta t
$$

Thus:

$$
S=\frac{Z_{1}}{Z_{1}-V \Delta t} .
$$

Extracting $\frac{Z_{1}}{V}$ from the equation above yields:

$$
T_{m}=\frac{Z_{1}}{V}=\frac{\Delta t}{S-1} .
$$

Equation 6 gives the momentary TTC solely on scalechange and time information.

\section{Modeling Acceleration}

The problem with the momentary TTC computation is that it neglects relative acceleration between the two vehicles. Relative acceleration will occur when the target vehicle performs a sudden stop or when the host vehicle is slowing down to avoid collision. Both cases are very important in an FCW application. Not detecting the host vehicle slowing down will give many false alarms (e.g. when nearing a stop light). Using the brake signal may not be enough since many times the driver might slow down by simply taking his or her foot off from the gas pedal.

Taking into account relative acceleration, the relative distance between the two vehicle as a function of time is given by:

$$
Z=Z_{0}+V_{0} \Delta t+\frac{1}{2} a \Delta t^{2}
$$

TTC (which we will denote simply as T) is the time that $Z=0$ (as in [4]), thus:

$$
T=\frac{-V_{0}+\sqrt{V_{0}^{2}-2 Z_{0} a}}{a}
$$

As mentioned above, the values distance, speed and acceleration are not natural vision measurements and we wish to use scale-change. In this section we show how to compute the actual TTC in a constant acceleration model based on $T_{m}$ and its derivative $\dot{T}_{m}$, both of which can be computed from scale-change in the image.

The momentary TTC is given by:

$$
T_{m}=-\frac{Z}{V}
$$


Thus its derivative is:

$$
\dot{T}_{m}=\frac{-\dot{Z} \cdot V+\dot{V} \cdot Z}{V^{2}}
$$

Since $\dot{Z}=V$ and $\dot{V}=a$ and we get:

$$
\dot{T}_{m}=\frac{-V^{2}+a \cdot Z}{V^{2}}=-1+\frac{a \cdot Z}{V^{2}}=\frac{a \cdot Z}{V^{2}}-1
$$

Let us define an assistance variable

$$
C=\dot{T}_{m}+1=\frac{a \cdot Z}{V^{2}}
$$

The $T_{m}$ and its derivative are measures taken from the current image thus $Z$ and $V$ actually refers to $Z_{0}$ and $V_{0}$, so we can say:

$$
T_{m}=-\frac{Z_{0}}{V_{0}}
$$

and

$$
C=\frac{a \cdot Z_{0}}{V_{0}^{2}} .
$$

Extracting $a$ from (14) we get:

$$
a=C \cdot \frac{V_{0}^{2}}{Z_{0}}
$$

Now we'll show how to define $T$ in the vision natural measures $T_{m}$ and $\dot{T}_{m}$. Substituting (15) in (8) we get:

$$
\begin{aligned}
T & =\frac{-V_{0}+\sqrt{V_{0}^{2}+2 C \cdot V_{0}^{2}}}{a} \\
& =\frac{-V_{0}+V_{0} \cdot \sqrt{1+2 C}}{a}
\end{aligned}
$$

Substituting (15) in (17) results in:

$$
\begin{aligned}
T & =\frac{-V_{0}+V_{0} \cdot \sqrt{1+2 C}}{C \cdot \frac{V_{0}^{2}}{Z_{0}}} \\
& =\frac{-1+\sqrt{1+2 C}}{C \cdot \frac{V_{0}}{Z_{0}}}
\end{aligned}
$$

Substituting $-T_{m}$ for $\frac{Z_{0}}{V_{0}}$ (equation 13 ) we get:

$$
\begin{aligned}
T & =\frac{-1+\sqrt{1+2 C}}{\frac{C}{-T m}} \\
& =-T_{m} \cdot \frac{-1+\sqrt{1+2 C}}{C} \\
& =T_{m} \cdot \frac{1-\sqrt{1+2 C}}{C}
\end{aligned}
$$

where $C$ is a function of $\dot{T}_{m}$ as in (12).

\section{Lateral Collision Decision}

After computing the TTC and determining that we are rapidly closing the distance to the target the second half of the FCW system goal is to determine if we are in fact on a possible collision course. In highway driving it is possible to use lane markings. In urban scenes lane markings may not exist and drivers may drive in-between lanes therefore this cue is not reliable. Fortunately the position of the vehicle boundaries in the image and their optic flow provide the required information.

We will first show that it is possible to determine a collision course based on image measurements alone without knowing the distance to the target vehicle or the target vehicles physical width. We will then show how to integrate the range information if it is known.

Let $x_{l}(t)$ and $x_{r}(t)$ be the image coordinates of the left and right edges of the target vehicle at time $t$. Let $Z(0)$ be the distance at time $t=0$ and we set $Z(0)=1$ in some arbitrary units. Using the perspective projection equation we can compute the vehicle width in the same arbitrary units:

$$
W=\frac{\left(x_{r}(0)-x_{l}(0)\right) Z(0)}{f} .
$$

As we approach the followed vehicle we can compute the range $Z(t)$ :

$$
\begin{aligned}
Z(t) & =\frac{f W}{x_{r}(t)-x_{l}(t)} \\
& =\frac{\left(x_{r}(0)-x_{l}(0)\right) Z(0)}{x_{r}(t)-x_{l}(t)}
\end{aligned}
$$

We can then use $Z(t)$ to compute the relative lateral position of the car:

$$
\begin{aligned}
& X_{l}(t)=\frac{x_{l}(t) Z(t)}{f} \\
& X_{r}(t)=\frac{x_{r}(t) Z(t)}{f}
\end{aligned}
$$

Substituting (24) into the above we get:

$$
\begin{aligned}
& X_{l}(t)=\frac{x_{l}(t) Z(0)}{f} \frac{x_{r}(0)-x_{l}(0)}{x_{r}(t)-x_{l}(t)} \\
& X_{r}(t)=\frac{x_{r}(t) Z(0)}{f} \frac{x_{r}(0)-x_{l}(0)}{x_{r}(t)-x_{l}(t)}
\end{aligned}
$$

Figure 2 a shows the results of tracking the left and right edge points of the followed vehicle as a function of time. These lines are then extrapolated to time $t=T T C$. If $X_{l}(t)$ is still to the left and $X_{r}(t)$ still to the right then the target vehicle is on a collision course. If both $X_{l}(t)$ and $X_{r}(t)$ are to one side then the target vehicle in not on a collision 


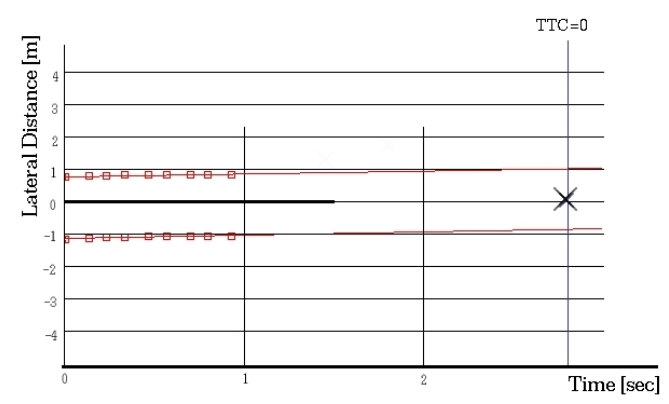

(a)

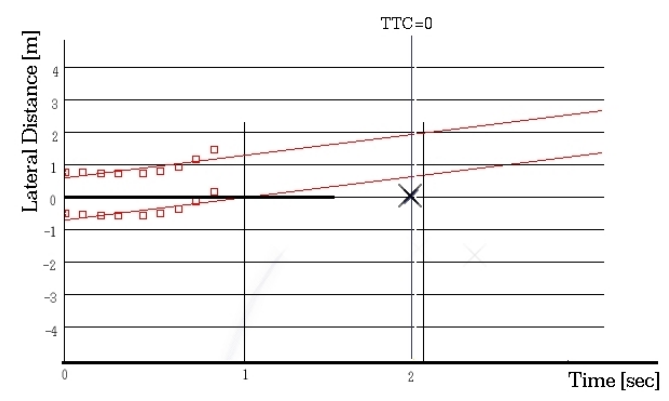

(b)

Figure 2: Results from balloon-car experiments. Lateral position of the outside edges of the target vehicle during a rapid approach plotted as a function of time ( $t)$. TTC is indicated by the tall vertical line and host vehicle position is marked by an X. (a) collision course. (b) one of the vehicles is performing an avoidance maneuver.

course with the camera mounted in the host vehicle. This is shown in figure $2 b$. The experiments were performed on a test track with a balloon-car as the target vehicle.

We use the last 9 measurements $(0.9 \mathrm{sec}$ at $10 \mathrm{HZ})$ and perform a linear fit. Since we only need to apply this procedure when the TTC is small $(T<3 \mathrm{sec})$ the extrapolation error is not large.

Note that the lateral positions $X_{l}(t)$ and $X_{r}(t)$ are in our arbitrary units. If we have even a rough estimate of $Z(0)$ then we convert the lateral position to meters and create a safety margin around the host vehicle. A method for computing the range estimate is shown in [2]

\section{Experiments and Results}

\subsection{Test Apparatus}

In normal driving conditions FCW rarely occurs and only false alarms can be tested in this way. In order to test FCW reliability and accuracy specific tests were performed in a test area.

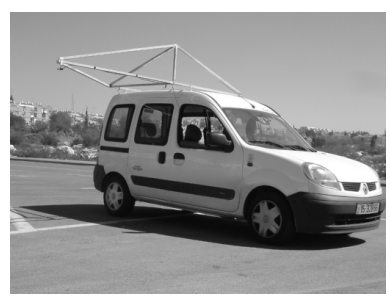

(a)

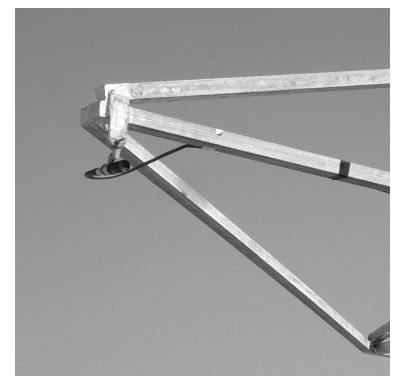

(b)
Figure 3: The remote mounting structure.

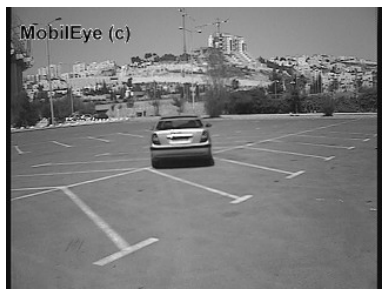

(a)

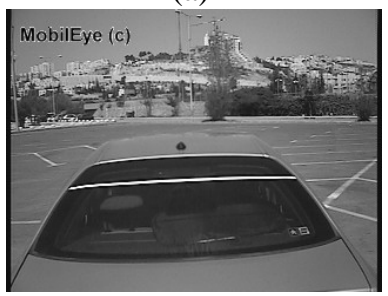

(c)

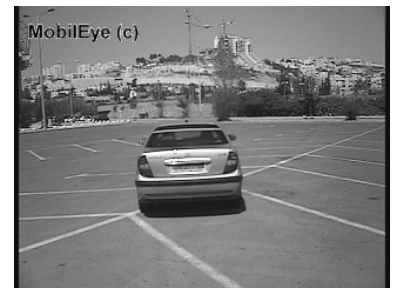

(b)

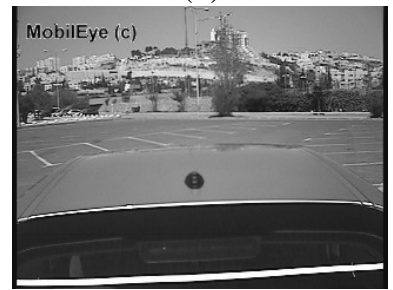

(d)
Figure 4: The camera passing just over the top of the target vehicle as recorded during a collision simulation. In (d) the camera is exactly above the rear bumper.

The tests had to simulate car crash, for this purpose a lightweight, rigid metal structure was built and was assembled on the top of the host vehicle (a Renault Kangoo), as shown in figure $3 \mathrm{a}$. The camera was mounted at the edge of this structure (see figure $3 \mathrm{~b}$ ) extending out to the right of the vehicle. The host vehicle can then pass to the left of the target vehicle with the remotely mounted camera passing just over the target vehicle a simulating a crash. The only difference is that the camera is about $20 \mathrm{~cm}$ higher than it would be if mounted inside the host vehicle. .

Figure 4 shows a crash simulation sequence. In order to know exactly the frame where the crash occured, a horizontal white line ribbon was marked on rear window of the target vehicle so that when the camera is exactly above the rear bumper of the target vehicle the white ribbon is at the bottom of the image (see figure 4d). 


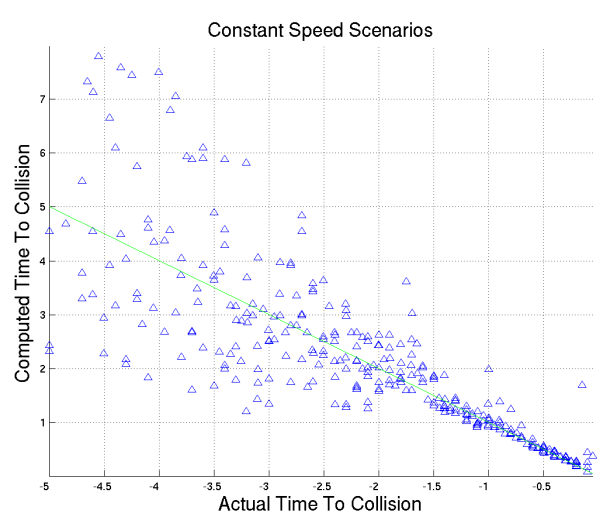

Figure 5: Scenario 1: constant relative speed. A comparison between the actual TTC (X-axis) and estimated TTC ( $Y$-axis) for all ten sequences.

\subsection{Accuracy Tests}

The first set of tests was TTC accuracy. An accuracy test was made by recording a collision sequence and then analyzing the TTC signal offline in the office. In this analysis the collision time is known and we can compare the predicted time to actual collision time.

There were two different scene dynamics measured: constant speed and relative acceleration:

1. Constant Relative Speed: the host vehicle drives toward a stationary target vehicle and past it at a constant speed.

2. Relative Acceleration: the host and lead vehicles drive at the same constant speed. The lead vehicle performs a sudden brake while the host vehicle continues in the same speed until passing the stopping (or stopped) lead vehicle.

Ten clips of each scenario were recorded. In figure 5 we see comparison between the real TTC and the estimated TTC for all ten clips of the constant speed experiments (scenario 1). We see that in general the estimated TTC is reasonably accurate below 2 seconds with the noise increasing when the TTC is larger.

In Figure 6 we see the results from one sequence from scenario 2: lead vehicle decelerating. We see that the momentary TTC is a curve while the TTC estimated using a constant acceleration model is correctly aligned aligned around the $y=-x$ line. In Figure 7 we see that this behavior is qualitatively the same for all the ten sequences.

In order to see quantitatively how the errors in TTC estimates depend on the actual TTC we binned the measurements into 1 second bins and computed the mean error and

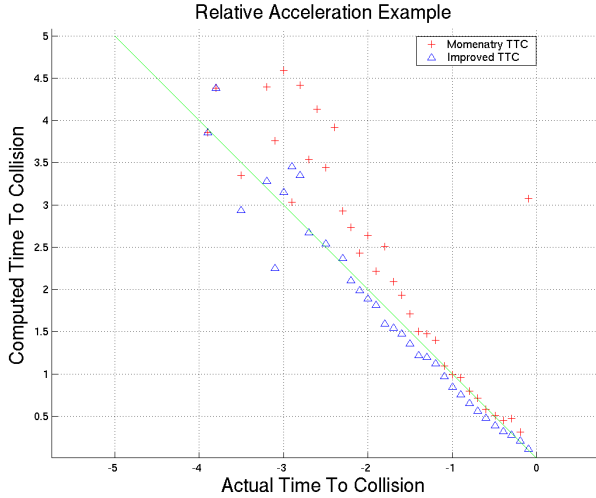

Figure 6: A single example of scenario 2: lead vehicle is decelerating. Comparing momentary TTC (crosses) and the modified TTC (triangles) to the ground truth TTC.

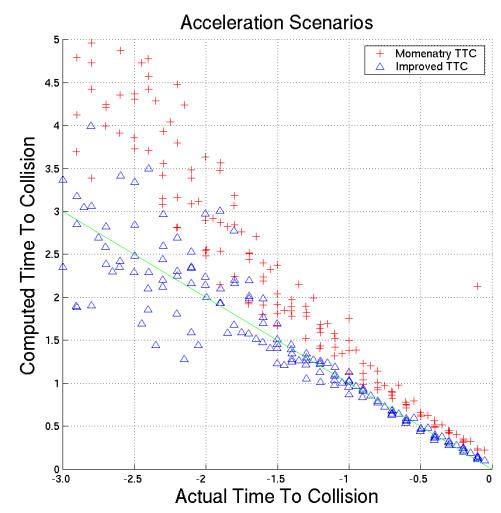

Figure 7: Scenario 2: lead vehicle is decelerating. Comparing momentary TTC (crosses) and the modified TTC (triangles) to the ground truth TTC for all 10 clips.

standard deviation for each bin separately. The results are shown in table 1. We see that for larger actual TTC the standard deviation of the estimated TTC increases. For TTC values below 2 seconds the TTC is quite accurate even in the presence of relative acceleration. Future work involves improving the tracking accuracy (namely scale measurement) to allow for reliable warnings at TTC of 3 seconds and up.

\subsection{Warning Tests}

In addition to measuring the signal quality, a series of tests was performed to test the effectiveness of the system. There were three types of scenarios: sudden brake avoidance, slow avoidance (slowly stopping behind a parking vehicle) and lateral avoidance. 


\begin{tabular}{|l|l|l|l|l|}
\hline TTC $(\mathrm{sec})$ & \multicolumn{2}{|c|}{ Constant Speed } & \multicolumn{2}{c|}{ Acceleration } \\
\hline \hline & mean & std & mean & std \\
\hline $0-1$ & 0.01 & 0.046 & 0.002 & 0.039 \\
\hline $1-2$ & -0.05 & 0.022 & -0.042 & 0.26 \\
\hline $2-3$ & -0.07 & 0.54 & -0.37 & 1.22 \\
\hline $3-4$ & 0.087 & 0.76 & -0.7 & 2.83 \\
\hline $4-5$ & -0.52 & 1.03 & N/A & N/A \\
\hline
\end{tabular}

Table 1: Errors in TTC estimates for the two tests scenarios: constant speed and relative acceleration. Results were binned according to the actual TTC and then the mean and standard deviation was computed for each bin.

\subsubsection{Sudden Brake Avoidance}

In this test we want to examine the relevance of the two second warning when the target (or lead) vehicle brakes suddenly. During these tests the two vehicles are traveling at $40 \mathrm{KPH}$ and the lead vehicle suddenly brakes. The driver of the host vehicle waits for the 2 second warning from the system and then performs an emergency braking. After both vehicles come to a complete stop we measured the relative position of the lead vehicle and the camera.

In the first set of ten tests the lead vehicle did a slow stopping (i.e. light braking so that it took approximately 4 seconds to reach a complete stop or approximately $0.3 \mathrm{~m} / \mathrm{s}^{2}$ ). In this test set the average distant after both vehicles were stopped was $2 m$ and the minimum distance $1 m$.

In the second set of tests the lead vehicle performed an emergency stop and came to a complete standstill in under 1 second. In this set the average distance after both vehicles were stopped was $30 \mathrm{~cm}$ and three out of ten sequences the camera passed the rear bumper of the lead car but by no more than $20 \mathrm{~cm}$. This means a collision at low speed as opposed to the $40 \mathrm{kph}$ collision without the system - a fender bender rather than serious injury.

\subsubsection{Slow Avoidance}

This test measured the false alarm rate when approaching a parked vehicle with the host vehicle driver aware and alert. This is a typical scenario when approaching a traffic light and the system must not produce a false signals in this case. The host vehicle is driving toward a stationary vehicle and slowly decelerate and fully stops behind the followed vehicle. In two out of the ten sequences a short false alarm was given each for two frames only ( 0.2 seconds). At the system level these false alarms can be eliminated using the host vehicles speedometer readings since the host vehicle is decelerating.

\subsubsection{Lateral Avoidance}

The host vehicle approaches the lead vehicle at a constant speed and when TTC is three seconds the host vehicle driver switches to the next lane to avoid crash. This is the typical situation of the host vehicle doing a very tight passing maneuver. This test was done with normal mounted camera inside the car and not with the test mount. In none of the ten tests a false warning was given.

\section{Summary and Future Work}

We have presented an FCW system which uses a single camera as input. Image scale change and image position are used directly for computing TTC and whether the target vehicle is on a collision path thus avoiding the need to compute distances and velocity. The system was tested on real-time hardware and the warning signal was shown to be useful in avoiding accidents or mitigating the consequences. Further improvements to the vehicle tracking are required to extend the warnings range to 3 seconds if desired.

\section{References}

[1] National Transportation Safety Board, Special Investigation Report - Highway Vehicle- and Infrastructure-based Technology For the Prevention of Rear-end Collisions. NTSB Number SIR-01/01, May 2001

[2] G. P. Stein, O. Mano and A. Shashua, Vision-based ACC with a Single Camera: Bounds on Range and Range Rate Accuracy In IEEE Intelligent Vehicles Symposium (IV2003),June 2003, Columbus, OH.

[3] G. R. Widman, W. A. Bauson and S. W. Alland Development of collision avoidance systems at Delphi Automotive Systems, In Proceedings of the Int. Conf. Intelligent Vehicles, pages $353-358,1998$

[4] L. Yang, J.H. Yang, E. Feron and V. Kulkarni development of a Performance-Based Approach for Rear-End Collision Warning and Avoidance System for Automobiles In IEEE Intelligent Vehicles Symposium (IV2003),June 2003, Columbus, $\mathrm{OH}$.

[5] P. Zador, S. Krawchuck and R. Voas Automotive Collision Avoidance System (ACAS) Program/First Annual Report. NHTSA, DOT HS 809 080, August 2000 